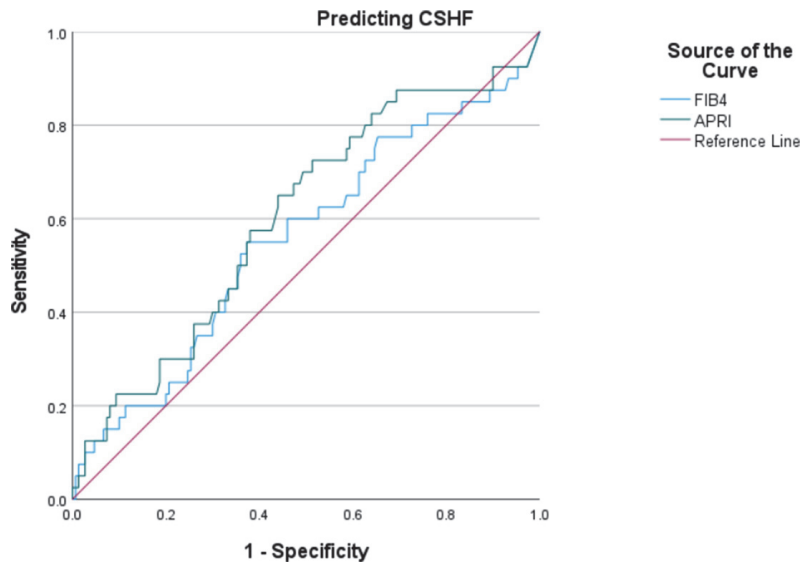

Abstract PWE-34 Figure 1 Predicting CSHF

(AUROC 0.556 and 0.603 respectively; Abstract PWE34 Figure 1).

Conclusions In these PLWH and elevated ALT, $\sim 20 \%$ had CSHF and $\sim 60 \%$ had HS. Lower HDL and diabetes were independent predictor of CSHF. Hazardous drinking or MS were identified in most patients with CSHF, however no risk factors were identified in almost 20\%. This raises the intriguing possibility that CSHF may be caused directly by the HIV infection.

\section{PWE-35 IMPROVED OUTCOMES FOLLOWING THE IMPLEMENTATION OF A DECOMPENSATED CIRRHOSIS DISCHARGE BUNDLE}

Katherine Smethurst*, Laura Jopson, Jennifer Gallacher, Titilope Majiyagbe, Amy Johnson, Philip Copeman, Stuart McPherson. Liver Unit, Newcastle Upon Tyne Hospitals NHS Foundation Trust, Newcastle Upon Tyne, UK

\subsection{6/gutjpl-2021-BSG.225}

Introduction Decompensated cirrhosis is a complex disorder with a high mortality rate and as a result re-admissions to hospital are common following discharge. Our aim was to evaluate the impact of implementation of a 'Decompensated Cirrhosis Discharge Bundle (DCDB)' and determine whether this improves the provision of evidence-based care and reduces preventable readmissions.

Methods A baseline review of the management of consecutive patients discharged with a diagnosis of decompensated cirrhosis was conducted in 2017 to assess the management of complications including ascites, encephalopathy, varices and alcohol misuse, and to determine readmission rates. Subsequently the DCDB was developed and implemented. Two cycles of evaluation of the impact of the bundle were conducted, the first using a paper version (Nov 2018-Oct 2019) and the second an electronic version (Nov 2020-March 2021).

Results Overall, 225 patients $(63 \%$ male; median age 55; median MELD 17; 72\% alcohol-related) were reviewed. Clinical and demographic features were similar in the 3 review periods. The overall 30 day readmission rate was 30\% (12\% potentially avoidable) in baseline review $(n=61)$ and areas for improvement were identified. In the first review following implementation of the DCDB $(n=86)$ only $23(27 \%)$ had a bundle completed. This increased to $69 \%(31 / 45)$ in the second review following implementation of the electronic DCDB. A comparison between patients with and without a DCDB is

\begin{tabular}{llll} 
Abstract PWE-35 Table 1 & & & \\
\hline & DCDB & No DCDB & $\mathrm{P}$ value \\
& $\mathrm{n}=54$ & $\mathrm{n}=138$ & \\
\hline Alcohol misuse & $63 \%$ & $64 \%$ & $\mathrm{p}=0.917$ \\
Alcohol team review & $85 \%$ & $66 \%$ & $\mathrm{p}=0.044$ \\
Thiamine prescribed & $91 \%$ & $85 \%$ & $\mathrm{p}=0.552$ \\
Community alcohol plan & $62 \%$ & $39 \%$ & $\mathrm{p}=0.026$ \\
HE related admission & $30 \%$ & $42 \%$ & $\mathrm{p}=0.138$ \\
Lactulose prescribed & $94 \%$ & $91 \%$ & $\mathrm{p}=1.0$ \\
Rifaximin prescribed & $94 \%$ & $84 \%$ & $\mathrm{p}=0.679$ \\
Ascites present & $70 \%$ & $69 \%$ & $\mathrm{p}=0.886$ \\
Discharge creatinine documented & $66 \%$ & $6 \%$ & $\mathrm{p}<0.001$ \\
Plan for U\&Es check after discharge & $61 \%$ & $36 \%$ & $\mathrm{p}=0.012$ \\
Variceal bleed & $15 \%$ & $11 \%$ & $\mathrm{p}=1.0$ \\
Beta-blockers, repeat OGD planned or TIPSS & $100 \%$ & $89 \%$ & $\mathrm{p}=0.526$ \\
Readmissions within 30 days & $31 \%$ & $25 \%$ & $\mathrm{p}=0.470$ \\
Potentially preventable liver related 30-day & $4 \%$ & $7 \%$ & $\mathrm{p}=0.407$ \\
readmission & & & \\
\hline
\end{tabular}

shown in the table 1 . Overall, use of the bundle was associated with improved care across all domains assessed.

Conclusions Implementation of a care bundle for patients with decompensated cirrhosis improved provision of evidence-based care at discharge. However, uptake of use of the bundle was slow but increased with an electronic version.

\section{PWE-36 TEMPORAL CHANGES IN THE PREVALENCE OF GALLBLADDER DYSPLASIA AND ADENOCARCINOMA IN PATIENTSUNDERGOING CHOLECYSTECTOMY}

${ }^{1}$ Wing Chou, ${ }^{1,2}$ Stephen Lam, ${ }^{3}$ Giles Toogood, ${ }^{2}$ Simon Wemyss-Holden, ${ }^{2}$ Alexia Tsigka, ${ }^{2}$ Bhaskar Kumar. ${ }^{1}$ Norwich Medical School, University of East Anglia, Norwich, UK; ${ }^{2}$ Norfolk and Norwich University Hospital NHS Trust, Norwich, UK; ${ }^{3}$ St. James's University Hospital, Hepatobiliary Unit, Leeds, UK

\subsection{6/gutjnl-2021-BSG.226}

Introduction A metaplasia-dysplasia-carcinoma sequence is the most plausible carcinogenic pathway for gallbladder cancer and although the incidence of gallbladder carcinoma is increasing, little is known about its precancerous lesions. The aim of this study was to determine temporal changes in the prevalence of low-grade dysplasia (LGD), high-grade dysplasia (HGD) and gallbladder adenocarcinoma and associated risk factors.

Methods We retrospectively identified consecutive patients who underwent cholecystectomy between January 2011 and March 2020. Patients were grouped according to histology: no dysplasia; LGD; HGD; and adenocarcinoma. Fitted linear models estimated temporal trends in prevalence and mean age for all histological outcomes. Logistic regression estimated associated risk factors.

Results A total of 5835 patients were included in the analysis. The prevalence of LGD was $1.47 \%$, HGD $0.17 \%$ and adenocarcinoma $0.19 \%$. Prevalence for all diseases increased over time, and mean age at diagnoses decreased over time. In a multivariate logistic regression model, with no dysplasia as the reference group, female sex increased the odds of LGD (OR 4.57, 95\% CI 3.07-10.10, $\mathrm{p}=<0.0001$ ). BMI was not associated with disease risk. 